\title{
Oportunidade perdida para diagnóstico oportunista de diabetes mellitus em comunidades quilombolas do sudoeste da Bahia, Brasil
}

\author{
Missed opportunity for timely diagnosis of diabetes mellitus \\ in Afrodescendant communities \\ in the southwest of the state of Bahia, Brazil
}

Cláudio Lima Souza ${ }^{1}$

Sabrina Martins Barroso ${ }^{2}$

Mark Drew Crosland Guimarães ${ }^{2}$

${ }^{1}$ Instituto Multidisciplinar em Saúde, Universidade Federal da Bahia. Av. Olivia Flores 3000, Universitário. 45.031-000 Vitória da Conquista BA Brasil. caulimas@gmail.com

${ }^{2}$ Programa de PósGraduação em Saúde Pública, Faculdade de Medicina, Universidade Federal de Minas Gerais.
Abstract The scope of this study was to estimate the prevalence and associated factors of missed opportunities for timely diagnosis of diabetes mellitus in Afrodescendant communities in Vitoria da Conquista in the state of Bahia. It involved a population-based cross-sectional study based on a probability sample $(n=797)$, where 548 individuals were eligible for analysis considering the risk criteria: age of 45 years old and above, increased Body Mass Index and hypertension. The prevalence of diabetes mellitus was estimated at $42.6 \%$. Logistic regression indicated the following statistically associated factors: self-assessed good/very good health conditions; not measuring blood pressure and not consulting with a physician in the past twelve months; and poor access to health services. The high prevalence was associated with factors related to low utilization and poor access to health services. Actions for the diagnosis and prevention of diabetes should prioritize populations at risk, including better education and training of family health care teams, and improvement of attendance at health care units. Enhanced integration between primary and secondary health care actions can positively impact early diagnosis, treatment and reduction of potential complications associated with diabetes in this population.

Key words Diabetes mellitus, Timely diagnosis, Afrodescendants
Resumo O objetivo deste estudo foi estimar prevalência e fatores associados com oportunidade perdida para diagnóstico oportunista de diabetes (PDO) em comunidades quilombolas de Vitória da Conquista, Bahia. Trata-se de um estudo de corte-transversal de base populacional em amostra probabilística $(n=797)$. Para esta análise 548 indivíduos foram elegíveis considerando os critérios de risco: idade igual ou superior a 45 anos, indice de massa corporal elevado e hipertensão arterial. A prevalência de PDO foi estimada em 42,6\%. Regressão logística demonstrou estarem estatisticamente associados, independentemente ao desfecho: autoavaliação de saúde boa/muito boa; não medir pressão arterial no último ano; não ter realizado consulta médica nos últimos doze meses; $e$, indice de acesso de serviços ruim. O elevado PDO apontado associou-se à baixa utilização e pior marcador de acesso aos serviços de saúde. Ações direcionadas ao diagnóstico e prevenção de DM devem priorizar populações de maior risco e incluir capacitação, treinamento das equipes de saúde da família e melhoria da estrutura e atenção nas unidades de saúde. Uma maior integração entre ações básicas de saúde e nível especializado pode impactar positivamente no diagnóstico precoce, e redução de possíveis complicações associadas a diabetes nesta população.

Palavras-chave Diabetes mellitus, Diagnóstico oportunista, Afrodescendentes 


\section{Introdução}

O Diabetes Mellitus (DM) é uma doença metabólica caracterizada por hiperglicemia resultante da secreção e/ou ação inadequada da insulina ${ }^{1}$. Constitui importante problema de saúde pública devido a sua alta carga de morbidade e mortalidade associadas. A Organização Mundial da Saúde (OMS) estima que 300 milhões de indivíduos desenvolverão DM até 2030 e 10 a 25\% apresentarão alguma comorbidade associada tais como doença cardiovascular, nefropatia, retinopatia e/ou neuropatia ${ }^{2}$. Estudo multicêntrico apontou prevalência de 7,6\% no Brasil, na década de $1980^{3}$. Mais recentemente, no ano de 2007, prevalências acima de $10 \%$ foram demonstradas na maioria dos Estados brasileiros ${ }^{4}$. Em 2011 o Brasil já possuía um contingente de 12 milhões de pessoas com DM com previsão de 20 milhões até $2030^{5}$. O DM constitui a primeira causa de mortalidade e hospitalizações juntamente com a hipertensão arterial ${ }^{6,7}$.

O DM pode cursar com um longo período assintomático dificultando ou retardando sua identificação. Contudo, há evidências de que o diagnóstico precoce e o tratamento adequado, especialmente a partir de 45 anos de idade, é custo-efetivo e contribui para a redução da mortalidade e de comorbidades ${ }^{8}$. O diagnóstico precoce de DM pode ser feito de forma ativa, por meio de programas de triagem ou inquéritos populacionais, ou de forma oportunista, quando pacientes buscam a atenção à saúde por outras razões 9 .

O exame de glicemia, utilizado no diagnóstico, é recomendado para todos os indivíduos com idade superior a 45 anos e indivíduos de qualquer idade com sobrepeso ou obesos que apresentem algum fator de risco adicional tais como inatividade física, parente de primeiro grau com DM, etnia afroamericana, latina ou indígena além de diagnóstico ou tratamento de hipertensão arterial. O exame deve ser repetido a cada três anos se o primeiro resultado for normal ${ }^{10}$. No entanto, a efetividade do diagnóstico oportunista depende de fatores relacionados ao sistema de saúde (e.g. oferta, organização, distribuição espacial das unidades prestadoras, disponibilidade de recursos) e de características do usuário (e.g. demográficas, socioeconômicas, culturais e psíquicas) ${ }^{11,12}$.

Dados sobre a falta de diagnóstico de DM demonstram a importância do tema. Estudo em área urbana do Brasil estimou que cerca de metade dos indivíduos com DM desconhece seu diagnóstico ${ }^{3}$. Nos Estados Unidos foi apontada prevalência de 40,0\% de perda de diagnóstico de
DM gestacional em população rural ${ }^{13}$. Etnias minoritárias, como uma comunidade de mexicanos-americanos, demonstrou prevalência de $51,3 \%$ de DM não diagnosticado ${ }^{14}$.

No Brasil, há poucos estudos de base populacional sobre o acesso e o diagnóstico oportunista de DM e nenhum conhecido em comunidades quilombolas. Esta população é caracterizada por ancestralidade negra e descendência de escravos, segundo critérios de autoatribuição étnico-racial. Apresentam trajetória histórica própria e são dotados de relações territoriais específicas ${ }^{15}$. Caracterizam-se também por viverem em contexto predominantemente rural com baixas condições socioeconômicas, e com baixo acesso aos serviços de saúde ${ }^{16-18}$.

As comunidades quilombolas já demonstram perfil de transição epidemiológica, convivendo hoje com doenças crônico degenerativas, como hipertensão e DM, mas ainda apresentando elevadas taxas de mortalidade infantil, desnutrição e doenças infecciosas ${ }^{16}$. A importância de estudar essa população é ressaltada por evidências de que minorias étnicas, como as populações afrodescendentes, apresentam maior risco para desenvolvimento de DM e suas complicações, além de menor acesso às ações de saúde ${ }^{19-21}$.

A atenção à saúde prestada às populações quilombolas está centrada nas Unidades Básicas de Saúde da Família (UBSF) e no trabalho do Agente Comunitário de Saúde (ACS). Cada UBSF é constituída por uma equipe multiprofissional cuja complexidade varia de acordo com a disponibilidade de recursos do município. A busca ativa de potenciais novos casos de DM, através da identificação de indivíduos apresentando fatores de risco durante as visitas domiciliares pelos ACS, é que possibilita o encaminhamento para avaliação e diagnóstico precoce dos pacientes, propiciando intervenção oportuna ${ }^{22}$. O diagnóstico oportunista de DM depende, portanto, dos diferentes níveis de atenção disponíveis nestas comunidades, incluindo o nível primário (básico), i.e., visita domiciliar, encaminhamento, consulta e solicitação do exame de glicemia, e o nível secundário, i.e., coleta de material e realização do exame em laboratório. Estudos demonstram que a realização da glicemia de jejum para triagem oportunista de DM tem se mostrado uma intervenção custo-efetiva ${ }^{8,23}$.

Apesar das evidências de maior risco para o DM, do limitado acesso e utilização de serviços de saúde e das precárias condições socioeconômicas de populações afrodescendentes, são escassas as informações sobre a saúde desta popu- 
lação, especialmente as referentes à utilização de serviços de maior complexidade. Estudo anterior da população quilombola de Vitória da Conquista indicou que $47,0 \%$ desta população tiveram pelo menos uma consulta médica nos últimos doze meses e 10,4\% dos indivíduos buscaram o serviço para realização de exames complementares ${ }^{18}$. Desta forma, o estudo da perda de diagnóstico oportunista de DM nas comunidades quilombolas poderá contribuir com o direcionamento de políticas públicas para redução do risco de desenvolvimento da doença através do diagnóstico precoce e consequente prevenção de comorbidades associadas. O presente artigo objetiva estimar a prevalência e estudar os fatores associados à perda de oportunidades para diagnóstico de DM em comunidades quilombolas, situadas na zona rural de Vitória da Conquista, Bahia, Brasil.

\section{Métodos}

\section{População}

Os dados apresentados nesta análise derivam de pesquisa intitulada Projeto COMQUISTA Comunidades quilombolas de Vitória da Conquista, Bahia: Avaliação de condicionantes de saú$\mathrm{de}^{18}$. Trata-se de estudo de corte transversal de base populacional, realizado em 2011 com amostra probabilística de comunidades quilombolas certificadas pela Fundação Palmares, adstritas ao município de Vitória da Conquista, Bahia. Este estudo foi aprovado pelos Comitês de Ética e Pesquisa da Faculdade São Francisco de Barreiras e da Universidade Federal de Minas Gerais.

O universo amostral foi estimado em 2.935 indivíduos adultos de 10 comunidades elegíveis. Para o cálculo da amostra, considerou-se: a) prevalência de $50 \%$; b) precisão de $5 \%$; c) nível de 95\% de confiança; d) efeito de desenho 2; e, e) $30 \%$ de perdas, totalizando 884 indivíduos adultos. Foram efetivamente entrevistados 797 indivíduos de cinco comunidades no inquérito principal. Para esta análise, 548 indivíduos foram elegíveis seguindo recomendações da Sociedade Brasileira de Diabetes (SBD) e da American Diabetes Association (ADA) para triagem de DM: idade maior que 45 anos ou idade inferior a 45 anos, mas apresentando, hipertensão arterial (PA igual ou maior a 140/90 mm Hg ou uso de anti-hipertensivo) e/ou IMC igual ou superior a $25 \mathrm{Kg} / \mathrm{m}^{2}$ (Figura 1) ${ }^{24,25}$.

Os dados foram obtidos por meio de entrevista domiciliar utilizando-se questionário semiestruturado da Pesquisa Nacional de Saúde ${ }^{26}$, adaptado e adequado ao contexto em linguagem, sequência e seleção das perguntas. Os dados foram coletados e armazenados em computadores portáteis (HP Pocket Rx5710 ${ }^{\mathrm{TM}}$ ), utilizando o programa QDS ${ }^{\mathrm{TM}}$ (Questionary Development System) versão 2.6.1. Para análise de confiabilidade, $4,5 \%$ das entrevistas foram repetidas, obtendose índices Kappa de concordância razoável a perfeita $(0,6 \text { a } 1,0)^{27}$.

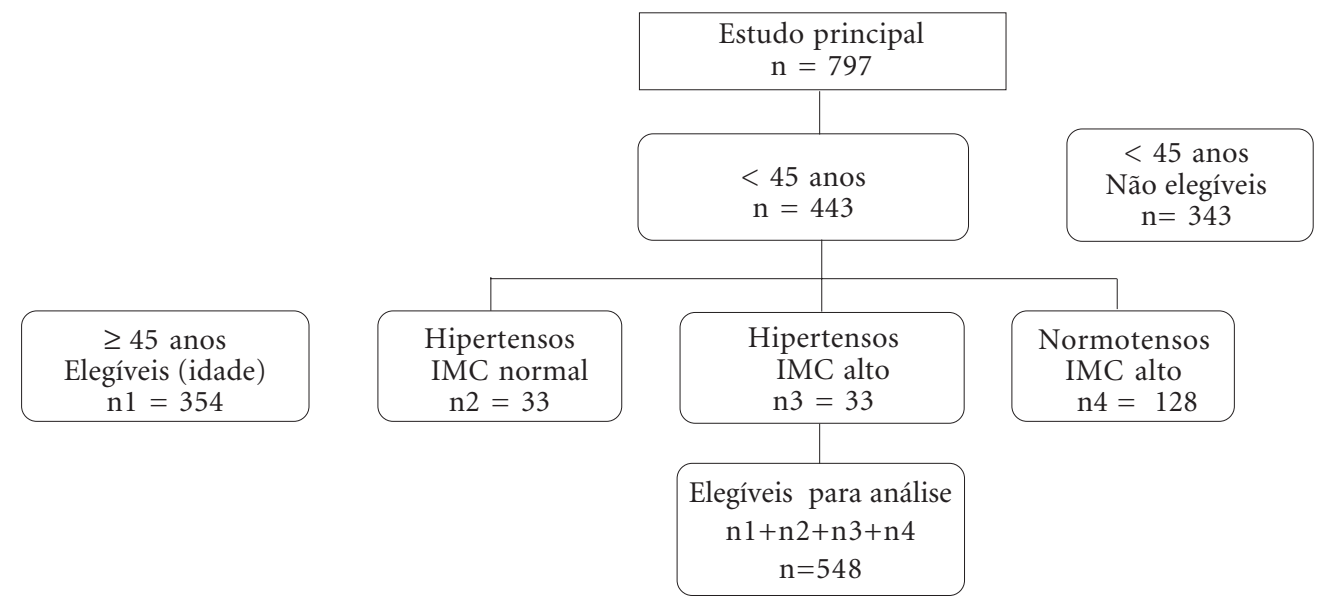

Figura 1. Critérios de definição da população para análise de oportunidades perdidas para diagnóstico oportunista de diabetes mellitus. 


\section{Evento e variáveis explicativas}

A variável dependente deste estudo foi a perda de oportunidade para diagnóstico oportunista de diabetes (PDO), definida como não ter realizado exame de glicemia DM ou tê-lo feito há mais de três anos. Tal medida foi avaliada por meio da pergunta "Quando foi a última vez que o(a) Sr.(a) fez exame de sangue para medir a glicemia, isto é, o açúcar no sangue?”.

As potenciais variáveis explicativas foram: 1) sociodemográficas (e.g. raça, autodefinição étnica, sexo, idade, estado conjugal, renda, escolaridade, tabagismo, autoavaliação do estado de saúde, prática de atividade física e etilismo);2) clínicas (e.g. história de doenças crônicas e medida de pressão arterial); e, 3) relacionadas ao serviço (e.g. cadastramento na UBSF, número de visitas do ACS, consulta médica nos últimos doze meses, medida de pressão arterial no último ano e índice de acesso aos serviços de saúde).

Para aferição das medidas antropométricas foram utilizados: balança eletrônica portátil Marte, modelo LC200pp ${ }^{\mathrm{TM}}$ com capacidade $200 \mathrm{~kg}$, para medida do peso; Estadiômetro portátil CAU$\mathrm{MAQ}^{\mathrm{TM}}$, modelo est-22A, para aferição da altura; e, esfigmomanômetro digital Omron HEM-742 ${ }^{\mathrm{TM}}$ para aferição pressão arterial. Todas as medidas aferidas segundo recomendações de padronização ${ }^{28}$. História de doenças crônicas refere-se ao relato de diagnóstico médico de pelo menos uma destas condições: hipertensão, hipercolesterolemia, cardiopatias, acidente vascular cerebral, bronquite asmática, artrite, dor crônica na coluna, tuberculose, depressão, outros transtornos mentais, doença pulmonar crônica e osteoporose. A autoavaliação da saúde foi agrupada em três categorias: boa/muito boa, regular, e ruim/muito ruim. Raça foi autorreferida segundo classificação do IBGE. Foi considerado uso de álcool o uso regular pelo menos uma vez por mês.

O índice de acesso aos serviços de saúde foi construído a partir da complexidade (presença ou carência de cada profissional) na equipe das UBSF das comunidades, o cadastramento do domicílio na UBSF e a frequência de visitas dos ACS aos domicílios. As equipes nas UBSF foram consideradas completas quando compostas por um médico, auxiliares de enfermagem, auxiliar de saúde bucal, dentista e enfermeiro, e foram consideradas incompletas quando contavam com auxiliares de enfermagem, dentista e enfermeiro. $\mathrm{O}$ acesso foi classificado em bom, razoável ou ruim, assim definidos: 1 . Bom - UBS com equipe completa, domicílio cadastrado na UBS e visitas men- sais do ACS ao domicílio; 2. Razoável - equipe profissional completa ou incompleta, domicílio cadastrado, uma a seis visitas anuais do ACS ao domicílio; e 3. Ruim - domicílio não cadastrado na UBS e/ou nenhuma visita domiciliar dos ACS nos últimos 12 meses, independente da equipe profissional estar completa ou incompleta.

\section{Análise estatística}

Foi conduzida análise descritiva por meio de distribuição de frequência de variáveis categóricas e medidas de tendência central das variáveis contínuas. A PDO foi estimada pela proporção de indivíduos que indicaram não ter realizado exame de glicemia ou tê-lo feito há mais de três anos em relação ao total de indivíduos com fatores de risco para DM. Para a análise univariada foi usado teste qui-quadrado, considerando 5\% de significância. A magnitude das associações entre as variáveis explicativas e o desfecho foi estimada pela Odds Ratio com intervalo de 95\% de confiança. Regressão logística foi realizada para identificação de fatores associados ao desfecho de forma independente. $\mathrm{O}$ modelo inicial incluiu variáveis com valor de $\mathrm{p}<0,20$ na análise univariada, permanecendo no modelo final apenas aquelas estatisticamente significativas $(\mathrm{p}<0,05)$. $\mathrm{O}$ ajustamento do modelo foi avaliado pelo teste de Hosmer e Lemeshow ${ }^{29}$. As análises foram realizadas utilizando os pacotes estatísticos $\mathrm{R}$ versão 2.11.1 e EPI INFO versões 3.5.1 e 7.0.9.7.

\section{Resultados}

Dos 548 indivíduos estudados (Figura 1), 231 $(42,6 \%)$ relataram não ter realizado teste de glicemia ou tê-lo realizado há mais de três anos, representando a perda de oportunidade para diagnóstico oportunista de diabetes (PDO). A maior parte da população estudada tinha 45 anos ou mais $(64,6 \%)$, relatou ter companheiro $(68,2 \%)$ e era composta por mulheres $(57,4 \%)$ (Tabela $1)$. Houve predomínio de pardos e pretos $(86,8 \%)$ e $83,9 \%$ dos indivíduos se autodefiniram como quilombolas. Metade da população recebia menos de meio salário mínimo e 40,7\% tinham menos de um ano de estudo. Nas questões relacionadas à saúde, 45,1\% dos indivíduos consideraram sua saúde regular, 40,6\% boa/muito boa e $14,3 \%$ ruim. Mais da metade da população $(52,9 \%)$ tinha níveis pressóricos superiores a $140 / 90 \mathrm{mmHg}$ ou usava medicamento anti-hipertensivo, sendo que $38,4 \%$ dos hipertensos não 
Tabela 1. Análise descritiva da população estudada para oportunidades perdidas, diagnosticando oportunistas para diabetes mellitus. Projeto Comquista, Brasil, 2011.

\begin{tabular}{|c|c|c|c|c|c|}
\hline Variável & $\begin{array}{c}\mathrm{N} \\
\text { total }^{\mathrm{a}}\end{array}$ & $\%$ & Variável & $\begin{array}{c}\mathrm{N} \\
\text { total }^{\mathrm{a}}\end{array}$ & $\%$ \\
\hline Faixa etária & & & Fuma atualmente & & \\
\hline $18-44$ anos & 194 & 35,4 & Não & 445 & 81,2 \\
\hline$\geq 45$ anos & 354 & 64,6 & $\operatorname{Sim}$ & 103 & 18,8 \\
\hline Sexo & & & Prática de atividade física últimos & & \\
\hline Feminino & 316 & 57,7 & 3 meses & & \\
\hline Masculino & 232 & 42,3 & Não & 446 & 81,4 \\
\hline Escolaridade & & & Sim & 102 & 18,6 \\
\hline Sem instrução & 221 & 40,7 & História de doenças crônicas & & \\
\hline 1 ou mais anos de estudo & 322 & 59,3 & Nenhuma & 265 & 48,4 \\
\hline Raça autorreferida & & & Pelo menos uma & 283 & 51,6 \\
\hline Branca & 69 & 12,8 & Hipertensão arterial ou uso & & \\
\hline Preta & 208 & 51,4 & de medicamento & & \\
\hline Amarela & 4 & 0,7 & anti-hipertensivo & & \\
\hline Parda & 249 & 46,2 & Não & 290 & 52,9 \\
\hline Indígena & 5 & 1,7 & Sim & 258 & 47,1 \\
\hline Autodefinição quilombola & & & Visita do Agente Comunitário & & \\
\hline Não & 92 & 17,1 & de Saúde & & \\
\hline Sim & 447 & 82,9 & Mensal & 260 & 60,7 \\
\hline Estado conjugal & & & 1 a 6 visitas anuais & 127 & 29,7 \\
\hline Sem companheiro & 174 & 31,8 & Nenhuma visita & 41 & 9,6 \\
\hline Com companheiro & 374 & 68,2 & Consulta médica nos últimos & & \\
\hline Renda & & & doze meses & & \\
\hline$<1 / 2$ Salário Mínimo b & 273 & 49,8 & Não & 234 & 42,7 \\
\hline$\geq 1 / 2$ Salário Mínimo ${ }^{\mathrm{b}}$ & 275 & 50,2 & Sim & 314 & 57,3 \\
\hline Autoavaliação do estado de saúde & & & Medida da $\mathrm{PA}^{\mathrm{c}}$ nos últimos & & \\
\hline Boa / Muito boa & 221 & 40,6 & doze meses & & \\
\hline Regular & 246 & 45,1 & Não & 118 & 21,9 \\
\hline Ruim / Muito ruim & 78 & 14,3 & Sim & 422 & 78,1 \\
\hline $\begin{array}{l}\text { Consumo de bebida alcoólica } \\
\text { pelo menos uma vez por mês }\end{array}$ & & & $\begin{array}{l}\text { Família cadastrada na Estratégia } \\
\text { de saúde da família }\end{array}$ & & \\
\hline Não & 355 & 64,8 & Não & 90 & 17,4 \\
\hline Sim & 193 & 35,2 & Sim & 428 & 82,6 \\
\hline
\end{tabular}

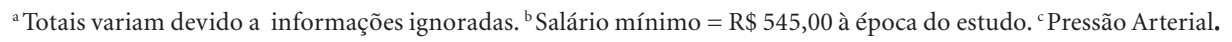

tinham realizado exame de glicemia. Os resultados mostraram ainda que entre os indivíduos que apresentaram IMC aumentado 41,7\% deixaram de realizar exame de glicemia. Em relação a hábitos e estilo de vida, $84,0 \%$ não praticaram atividade nos últimos três meses, 35,2\% relataram uso de álcool ao menos uma vez por mês e $18,8 \%$ fumavam diariamente ou eventualmente.

O índice de acesso aos serviços foi avaliado como ruim em 28,4\% dos indivíduos. Das cinco comunidades estudadas, uma não tinha equipe de saúde completa na época do estudo, correspondendo a $17,4 \%$ de indivíduos que eram assistidos por equipe de saúde incompleta. Apesar de $82,6 \%$ das famílias estarem cadastradas nas
UBSF, 38,5\% recebiam visita mensal do ACS e $9,6 \%$ não receberam visita no último ano. Ainda que visitados pelos ACS, 42,7\% não haviam consultado o médico nos últimos doze meses, mesmo com o relato de $51,6 \%$ dos indivíduos com diagnóstico prévio de alguma doença crônica.

A análise univariada indicou que estiveram associadas com PDO as seguintes variáveis (Tabela 2): faixa etária 18-44 anos, autoavaliação de saúde boa/muito boa, relato de não ter história de doença crônica diagnosticada, não ter aferido a pressão arterial no último ano $(\mathrm{OR}=3,67, \mathrm{p}<$ $0,001)$, não ter realizado consulta médica nos últimos 12 meses $(\mathrm{OR}=4,27, \mathrm{p}<0,001)$ e não estar cadastrado na UBSF (OR 2,21, p =0,002). Além 
Tabela 2. Análise univariada de oportunidades perdidas para diagnóstico oportunista de diabetes mellitus em comunidades quilombolas de Vitória da Conquista. Projeto Comquista, Bahia, Brasil, 2011.

\begin{tabular}{|c|c|c|c|c|}
\hline Variável & $\mathbf{N}$ & $\operatorname{PDO}(\%)^{a}$ & OR (IC 95\%) & Valor de $\mathrm{p}^{\mathrm{c}}$ \\
\hline \multicolumn{5}{|l|}{ Faixa etária } \\
\hline $18-44$ anos & 194 & $104(53,6)$ & $2,02(1,41-2,87)$ & $<0,001^{\mathrm{c}}$ \\
\hline$\geq 45$ anos & 348 & $127(36,5)$ & 1 & \\
\hline \multicolumn{5}{|l|}{ Sexo } \\
\hline Feminino & 310 & $128(41,3)$ & 1 & \\
\hline Masculino & 232 & $103(44,4)$ & $0,88(0,63-1.24)$ & 0,47 \\
\hline \multicolumn{5}{|l|}{ Escolaridade } \\
\hline Sem instrução & 221 & $96(43,4)$ & $1,07(0,76-1,51)$ & 0,70 \\
\hline 1 ou mais anos de estudo & 316 & $132(41,8)$ & 1 & \\
\hline \multicolumn{5}{|l|}{ Estado conjugal } \\
\hline Sem companheiro & 169 & $87(51,5 \%)$ & $1,69(1,17-2,43)$ & $0,005^{c}$ \\
\hline Com companheiro & 373 & $144(38,6)$ & 1 & \\
\hline \multicolumn{5}{|l|}{ Renda individual mensal } \\
\hline < 1/2 Salário Mínimo ${ }^{\text {b }}$ & 272 & $123(45,2)$ & $1,24(0,88-1,74)$ & 0,22 \\
\hline$\geq 1 / 2$ Salário Mínimo ${ }^{\mathrm{b}}$ & 270 & $108(40,0)$ & 1 & \\
\hline \multicolumn{5}{|l|}{ Trabalha atualmente } \\
\hline Não & 295 & $113(38,3)$ & $0,68(0,49-0,96)$ & $0,03^{c}$ \\
\hline Sim & 247 & $118(47,8)$ & 1 & \\
\hline \multicolumn{5}{|c|}{ Autoavaliação do estado de saúde } \\
\hline Boa / Muito boa & 218 & $112(51,4)$ & $2,64(1,51-4,63)$ & $<0,001^{c}$ \\
\hline Regular & 245 & $97(39,6)$ & $1,64(0,94-2,86)$ & 0,08 \\
\hline Ruim / Muito ruim & 77 & $22(28,6)$ & 1 & \\
\hline \multicolumn{5}{|c|}{ Prática de atividade física últimos 3 meses } \\
\hline Não & 441 & $194(44,0)$ & $1,36(0,87-2,12)$ & 0,18 \\
\hline $\operatorname{Sim}$ & 101 & $37(36,6)$ & 1 & \\
\hline \multicolumn{5}{|l|}{$\begin{array}{l}\text { Consumo de bebida alcoólica } \\
\text { pelo menos uma vez por mês }\end{array}$} \\
\hline Sim & 193 & $90(46,6)$ & $0,78(0,54-1,11)$ & 0,16 \\
\hline Não & 349 & $141(40,4)$ & 1 & \\
\hline \multicolumn{5}{|l|}{ Tabagismo } \\
\hline Sim & 103 & $49(47,6)$ & $0,78(0,51-1,20)$ & 0,26 \\
\hline Não & 439 & $182(41,5)$ & 1 & \\
\hline \multicolumn{5}{|l|}{ História de doenças crônicas } \\
\hline Nenhuma & 264 & $142(53,8)$ & $2,47(1,74-3,51)$ & $<0,001^{\mathrm{c}}$ \\
\hline Pelo menos uma & 278 & $89(32,0)$ & 1 & \\
\hline \multicolumn{5}{|c|}{ Aferição da $\mathrm{PA}^{\mathrm{d}}$ nos últimos doze meses } \\
\hline Não & 118 & $79(66,9)$ & $3,67(2,38-5,66)$ & $<0,001^{\mathrm{c}}$ \\
\hline Sim & 419 & $149(35,6)$ & 1 & \\
\hline \multicolumn{5}{|c|}{ Consulta médica nos últimos 12 meses } \\
\hline Não & 233 & $145(62,2)$ & $4,27(2,97-6,15)$ & $<0,001^{\mathrm{c}}$ \\
\hline Sim & 309 & $86(27,8)$ & 1 & \\
\hline \multicolumn{5}{|c|}{ Cadastro na Unidade de Saúde da Família } \\
\hline Não & 76 & $42(55,3)$ & $2,21(1,34-3,66)$ & $0,002^{\mathrm{c}}$ \\
\hline $\operatorname{Sim}$ & 349 & $125(35,8)$ & 1 & \\
\hline \multicolumn{5}{|c|}{ Visitas do agente comunitário ao domicílio } \\
\hline Nenhuma & 40 & $17(42,5)$ & $0,85(0,43-1,67)$ & 0,63 \\
\hline 1 a 6 visitas anuais & 125 & $53(42,4)$ & $0,85(0,55-1,31)$ & 0,47 \\
\hline Mensal & 257 & $99(38,5)$ & 1 & \\
\hline \multicolumn{5}{|c|}{ Índice de acesso aos serviços de saúde } \\
\hline Acesso bom & 210 & $76(36,2)$ & 1 & \\
\hline Acesso razoável & 117 & $49(41,9)$ & $1,27(0,80-2,02)$ & 0,16 \\
\hline Acesso ruim & 130 & $66(50,8)$ & $1,82(1,17-2,83)$ & $0,008^{c}$ \\
\hline
\end{tabular}

${ }^{a}$ Número e proporção de oportunidade perdida para diagnóstico oportunista para cada categoria. ${ }^{\text {b }}$ Salário mínimo à época do estudo R \$ 545,00. ' Significância estatística - p $<0.05$ em teste qui-quadrado. 
disto, o índice de acesso a serviços apresentou relação inversa com PDO - quanto pior o índice maior a perda de oportunidade de diagnóstico. Indivíduos com acesso precário apresentaram PDO de 50,8\% (OR 2,03 p = 0,008).

O modelo logístico multivariado final (Tabela 3 ) indicou que as seguintes variáveis estiveram independentemente associadas à $\mathrm{PDO}(\mathrm{p}<0,05)$ : autoavaliação de saúde boa/muito boa, não ter tido a pressão arterial aferida no último ano, não ter realizado consulta médica nos últimos doze meses e possuir índice de acesso aos serviços de saúde ruim. O teste de adequação de HosmerLemeshow apontou para um bom ajustamento do modelo final $(\mathrm{p}=0,49)$.

\section{Discussão}

A perda de oportunidade para diagnóstico oportunista de DM nesta população foi de $42,6 \%$, e corroboram com dados de outros estudos sobre perda de diagnóstico de DM. Zhang et al. ${ }^{30}$ indicaram prevalência de $42,0 \%$ de DM não diagnosticado nos Estados Unidos, enquanto FisherHoch et al. ${ }^{14}$ demonstraram $50,0 \%$ de perda de diagnóstico em população mexicano-americana. Maior prevalência e risco de $\mathrm{DM}$ em populações negras já foram demonstradas ${ }^{31}$, com potencialmente maior perda de oportunidade de diagnóstico. Nossos resultados nas comunidades quilombolas apontaram uma proporção de DM autorreferida de $8,2 \%$, (dados não apresentados), superior à média brasileira de $5,6 \%^{32}$.

Indivíduos com idade inferior a 45 anos apresentaram maior prevalência de PDO. Kaufmann et al. ${ }^{13}$ destacaram menor propensão a diagnós- tico em indivíduos jovens por menor preocupação e envolvimento com ações de saúde. Foi observada alta prevalência de hipertensão nesta faixa etária $(30,7 \%)$, dos quais 55,0\% não realizaram exame de glicemia. Estes dados alertam para a importância da avaliação de jovens, embora não sejam alvos preferenciais para diagnóstico de DM, as recomendações do MS incluem o exame de glicemia em indivíduos hipertensos, independente da faixa etária ${ }^{33}$.

Chama atenção os baixos níveis de renda e escolaridade desta população, indicando seu alto grau de pobreza e vulnerabilidade social. Apesar disto, estas variáveis não se mostraram associadas à PDO nesta análise, o que pode ser parcialmente explicado pelo baixo poder discriminativo destas características considerando a homogeneidade desta população em pobreza extrema. No entanto, estes resultados são corroborados por Wilder et al. ${ }^{34}$ que verificaram como determinantes para DM não diagnosticado na população norte-americana a falta de acesso aos cuidados de saúde em detrimento às variáveis de renda e escolaridade.

Uma autoavaliação de saúde boa/muito boa esteve associada a maior PDO o que é explicado pela menor procura aos serviços de saúde quando se percebe boa saúde, principalmente em se tratando de diagnóstico oportunista que depende da presença do indivíduo no serviço.

As variáveis relacionadas ao serviço de saúde foram as que estiveram mais fortemente associadas à PDO nas comunidades estudadas. Os registros sobre as comunidades deste estudo indicavam $100,0 \%$ de cobertura prevista da Estratégia de Saúde da Família, no entanto $86,0 \%$ dos domicílios participantes do estudo relataram es-

Tabela 3. Análise multivariada para oportunidades perdidas de triagem diagnóstica para diabetes mellitus. Projeto Comquista, Bahia, Brasil, 2011.

\begin{tabular}{lcc}
\hline \multicolumn{1}{c}{ Variável } & Odds Ratio (IC 95\%) & ${\text { Valor de } \mathbf{p}^{\mathbf{a}}}$ \\
\hline Autoavaliação do estado de saúde & 1 & \\
$\quad$ Ruim / Muito ruim & $1,28(0,81-2,00)$ & 0,29 \\
$\quad$ Regular & $2,69(1,35-5,34)$ & $0,005^{\mathbf{a}}$ \\
$\quad$ Muito Bom/ Bom & $2,15(1,27-3,63)$ & $<0,005^{\mathbf{a}}$ \\
Não medir pressão arterial nos últimos doze meses & $3,48(2,26-5,36)$ & $<0,001^{\mathbf{a}}$ \\
Não consultar médico nos últimos 12 meses & 1 & 0,26 \\
Índice de acesso ao serviço de saúde & $1,37(0,79-2,38)$ & $0,034^{\mathbf{a}}$ \\
$\quad$ Acesso bom & $1,71(1,04-2,81)$ & \\
Acesso razoável & & \\
Acesso ruim & &
\end{tabular}

${ }^{a}$ Valores significantes $(\mathrm{p}<0,05)$. Teste de adequação do modelo - Hosmer Lemeshow $\mathrm{p}=0,49$. 
tar cadastrados. O acompanhamento das UBSF possibilitariam a busca ativa e o diagnóstico oportunista em assintomáticos por meio da avaliação dos fatores de risco. No entanto, verificase um quadro preocupante com relação ao acesso e utilização dos serviços nestas comunidades, confirmado pela associação independente entre o índice de acesso ruim e a PDO (OR 1,71, p < $0,05)$. Este índice reflete que equipes de saúde incompletas, número insuficiente de visitas do ACS e falta de cadastramento na UBSF são importantes marcadores de perda de oportunidade para diagnóstico oportunista. Além disso, a presença do médico na equipe, caracterizando melhor índice de acesso, pode ser fator relevante para este resultado. Isto pode ser corroborado pela maior chance de PDO $(\mathrm{OR}=3,48)$ nos indivíduos que não realizaram consulta médica nos últimos doze meses. O médico, como profissional que solicita exame complementar e prescreve, torna-se ator fundamental na atenção secundária aos indivíduos encaminhados por ACS ou que aparecem espontaneamente para consultas por outros motivos. Por outro lado, a associação entre PDO e não ter tido a pressão arterial aferida nos últimos doze meses $(\mathrm{OR}=2,15)$ é preocupante, pois reflete importante ato que outros profissionais da ESF estão aptos desenvolver, inclusive durante as visitas domiciliares e que pode não estar sendo adequadamente realizado. A não aferição da pressão arterial, além de não propiciar diagnóstico de potencial hipertensão arterial, não permite a identificação de um dos mais importantes fatores associados com DM, prejudicando assim o encaminhamento oportuno. Os dados apresentados de cobertura incompleta, não aferição da pressão arterial e um índice de acesso aos serviços ruim são corroborados por outros estudos que confirmam a existência de importantes barreiras para as populações minoritárias na busca e obtenção de atenção à saúde ${ }^{14,18,23}$. As perdas são denotadas desde o cadastramento na UBSF, encaminhamento pelo ACS, realização de consulta médica, solicitação e realização de exames laboratoriais, com impacto imediato na prevalência de PDO. Para minimizar a perda de diagnóstico oportunista de DM é preciso que a equipe de saúde esteja completa, capacitada e treinada para a identificação dos fatores de risco associados à doença, além da necessida- de de regularidade nas visitas domiciliares realizadas pelos ACS.

O estudo apresenta algumas limitações. As informações sobre realização dos exames são autorreferidas, podendo ser afetadas por memória ou conhecimento, embora sua confiabilidade já esteja bem demonstrada na população brasileira ${ }^{35}$. O delineamento seccional limita a interpretação das associações de causa e efeito e o questionário não contemplou histórico familiar de DM, podendo potencialmente causar uma subestimação da já elevada prevalência de PDO encontrada.

Apesar disto, a alta prevalência de PDO neste estudo indica um potencial déficit na abordagem para diagnóstico oportunista de DM e, consequentemente, um impacto negativo na prevenção da doença e comorbidades associadas. Ter DM não diagnosticada e desenvolver morbidades traz importantes riscos à saúde além de representar elevados custos financeiros aos serviços de saúde em longo prazo $^{30}$. Considerando o diagnóstico precoce mais custo-efetivo que o tratamento das complicações ${ }^{5,8}$, e sabendo que o status de minoria confere um risco duas vezes maior de ter DM além de maior risco de complicações $^{36}$, nossos resultados apontam para a necessidade de se intensificar as ações voltadas ao diagnóstico oportunista de DM nas comunidades estudadas.

Estratégias podem ser desenvolvidas para diminuir a PDO nestas comunidades incluindo melhoria do acesso a exames laboratoriais - pela oferta de unidades de coleta de sangue próximas às comunidades; intensificação das ações de treinamento das equipes de saúde da família para abordagem de populações que apresentem maior risco de desenvolvimento de DM; capacitação dos ACS para, durante as visitas domiciliares, avaliar a presença de fatores de risco já estabelecidos, podendo assim encaminhar para investigação oportuna nos serviços de referência. Uma estratégia fundamentada na continuidade e integralidade entre a atenção básica e a secundária poderá proporcionar uma maior efetividade das ações de atenção e prevenção do DM em comunidades quilombolas, predominantes rurais e em extrema desigualdade social, buscando assim a desejada equidade prevista nos fundamentos do Sistema Único de Saúde. 


\section{Colaboradores}

CL Souza e MDC Guimarães trabalharam na concepção, análise do assunto, revisão da literatura, análises estatísticas, redação e revisão do texto; SM Barroso trabalhou na concepção, redação, revisão da literatura e revisão do texto.

\section{Referências}

1. American Diabetes Association. Diagnosis and classification of Diabetes mellitus. Diabetes Care 2013; 36(Supl. 1):567-574

2. Organização Mundial da Saúde (OMS). Adherence to long-term therapies - evidence for action, 2003. [acessado 2013 maio 28]. Disponível em: http://www. who.int/chp/knowledge/publications/adherence_ full_report.pdf

3. Malerbi D, Franco LJ. The Brazilian Cooperative Group on the Study of Diabetes Prevalence. Multicenter Study of the Prevalence of Diabetes Mellitus and Impaired Glucose Tolerance in the urban Brazilian population aged 30-69 years. Diabetes Care 1992; 15(11):1509-1516.

4. Dias JCR, Campos JADB. Diabetes mellitus: reasons for prevalence in different geographic regions of Brazil, 2002-2007. Cien Saude Colet 2012; 17(1):239244.

5. Schmidt MI, Duncan BBE, Silva GA, Menezes AM, Monteiro CA, Barreto SM, Chor D, Menezes PR. Chronic non-communicable diseases in Brazil: burden and current challenges. Lancet 2011; 377(9781):1949-1961.

6. Wild S, Roglic G, Green A, Sicree RE, King H. Global Prevalence of Diabetes - Estimates for the year 2000 and projections for 2030. Diabetes Care 2004; 27(5):1047-1053.

7. Brasil. Ministério da Saúde (MS). Cadernos de Atenção Básica, no 16 - Diabetes Mellitus. Brasília: MS; 2006. [acessado 2013 maio 28]. Disponível em: http:/ /bvsms.saude.gov.br/bvs/publicacoes/diabetes_ mellitus.PDF

8. Li R, Zhang P, Barker LE, Chowdhury FM, Zhang $X$. Cost-effectiveness of interventions to prevent and control diabetes mellitus: a systematic review. $D i-$ abetes Care 2010; 33(8):1872-1894.

9. Ealovega MW, Tabaei BP, Brandle M, Burke R, Herman WH. Opportunistic screening for diabetes in routine clinical practice. Diabetes Care 2004; 27(1):912.

10. American Diabetes Association. Standards of Medical Care in Diabetes. Diabetes Care 2011; 34(Supl. 1):S11-S61.

11. Travassos C, Martins M. Uma revisão sobre os conceitos de acesso e utilização de serviços de saúde Cad Saude Publica 2004; 20(Supl. 2):190-198.

12. Unglert CVS, Rosenburg CP, Junqueira CB. Access to health services: a geographical approach to public health. Rev Saude Publica; 1987; 21(5):439-446.

13. Kaufmann RC, Smith T, Bochantin T, Khardori R, Evans MS, Steahly L. Failure to obtain follow-up testing for gestational diabetic patients in a rural population. Obstet Gynecol 1999; 93(5 Pt 1):734-737.

14. Fisher-Hoch SP, Vatcheva KP, Laing ST, Hossain MM, Rahbar MH, Hanis CL, Brown HS 3rd, Rentfro AR, Reininger BM, McCormick JB. Missed Opportunities for Diagnosis and Treatment of Diabetes, Hypertension, and Hypercholesterolemia in a Mexican American Population, Cameron County Hispanic Cohort, 2003-2008. Prev Chronic Dis 2012; 9:110298. 
15. Brasil. Decreto no 4887, de 20 de novembro de 2003. Regulamenta o procedimento para identificação, reconhecimento, delimitação, demarcação e titulação das terras ocupadas por remanescentes das comunidades dos quilombos de que trata o art. 68 do Ato das Disposições Constitucionais Transitórias. Diário Oficial da União 2003; 21 nov.

16. Volochko A. A saúde nos Quilombos. In: Volochko A, Batista LE. Saúde nos Quilombos. São Paulo: Instituto de Saúde 2009; p. 147-168. (Temas em Saúde Coletiva, 9).

17. Silva MJG, Lima FSS, Hamann EM. Uso dos serviços públicos de saúde para DST/HIV/AIDS por comunidades remanescentes de quilombos no Brasil. Saude Soc 2010; 19(Supl. 2):109-120.

18. Gomes KO, Reis EA, Guimarães MDC, Cherchiglia ML. Utilização de serviços de saúde por população quilombola do Sudoeste da Bahia, Brasil. Cad Saude Publ. No prelo 2013.

19. Oldroyd J, Banerjee M, Heald A, Cruickshank K. Diabetes and ethnic minorities. Postgrad Med J 2005; 81(958):486-490.

20. Lopes F, Buchalla CM, Ayres JRCM. Black and nonBlack women and vulnerability to HIV/AIDS. Rev Saude Publ 2007; 41(Supl. 2):39-46.

21. Leal MC, Gama SGN, Cunha CB. Desigualdades raciais, sociodemográficas e na assistência ao pré-natal e ao parto. Município do Rio de Janeiro - Brasil, 1999-2001. Rev Saude Publ 2005; 39(1):100-107.

22. Silva ARV, Zanetti ML, Forti AC, Freitas RWJF, Hissa MN, Damasceno MMC. Avaliação de duas intervenções educativas para a prevenção do Diabetes Mellitus tipo 2 em adolescentes. Texto \& Contexto - Enfermagem 2011; 20(4):782-787.

23. Sheehy A, Pandhi N, Coursin DB, Flood GE, Kraft SA, Johnson HM, Smith MA. Minority status and diabetes screening in an ambulatory population. Diabetes Care 2011; 34(6):1289-1294.

24. American Diabetes Association. Standards of medical care in diabetes -2013. Diabetes Care 2013; 36(Supl. 1):S15.

25. Sociedade Brasileira de Diabetes (SBD). Tratamento e acompanhamento do Diabetes Mellitus, Diretrizes da Sociedade Brasileira de Diabetes. 3a ed. Itapevi: A. Araújo Silva Farmacêutica; 2009.

26. Pesquisa Nacional de Saúde (PNS). Inquérito Região Integrada do Distrito Federal (RIDE/DF). [acessado 2011 abr 2]. Disponível em: http://www.pns. icict.fiocruz.br/index.php?pag=proposicao
27. Szklo M, Nieto JF. Epidemiology: beyond the basics. $2^{\text {nd }}$ ed. Baltimore: Aspen Publishers; 2007.

28. Barreto SM, Passos VM, Firmo JO, Guerra HL, Vidigal PG, Lima-Costa MF. Hypertension and clustering of cardiovascular risk factors in a community in Southeast Brazil: the Bambuí Health and Ageing Study. Arq Bras Cardiol 2001; 77(6):576-581

29. Hosmer DW, Lemeshow S. Applied logistic regression. New York: Wiley-interscience Publication; 1989.

30. Zhang X, Geiss LS, Cheng YJ, Beckles GL, Gregg EW, Kahn HS. The missed patient with diabetes: how access to health care affects the detection of diabetes. Diabetes Care 2008; 31(9):1748-1753.

31. Oliveira F. Saúde da população negra: Brasil ano 2001. Brasília: Organização Pan-Americana da Saúde (OPAS); 2002.

32. Brasil. Ministério da Saúde (MS). Vigitel Brasil 2011: vigilância de fatores de risco e proteção para doenças crônicas por inquérito telefônico. Dados sobre Diabetes. Brasília: MS; 2011. [acessado 2014 abr 19]. Disponível em: http://bvsms.saude.gov. br/bvs/publicacoes/ vigitel_brasil_2011_fatores_risco_doencas_cronicas. pdf

33. Sociedade Brasileira de Cardiologia, Sociedade Brasileira de Hipertensão, Sociedade Brasileira de Nefrologia. VI Diretrizes Brasileiras de Hipertensão. Arq Bras Cardiol 2010; 95(Supl. 1):1-51.

34. Wilder RP, Majumdar SR, Klarenbach SW, Jacobs P. Socio-economic status and undiagnosed diabetes. Diabetes Res Clin Pract 2005; 70(1):26-30.

35. Dachs NW. Determinantes das desigualdades na auto-avaliação do estado de saúde no Brasil: análise dos dados da PNAD/ 98. Cien Saude Colet 2002; 7(4):641-670.

36. Sheehy A, Pandhi N, Coursin DB, Flood GE, Kraft SA, Johnson HM, Smith MA. Minority status and diabetes screening in an ambulatory population. Diabetes Care 2011; 34(6):1289-1294.

Artigo apresentado em 06/06/2013

Aprovado em 13/07/2013

Versão final apresentada em 16/07/2013 